\title{
ASSESSMENT OF THE PREVALENCE INDEX ON SIGNS OF COMBINATION SYNDROME IN PATIENTS TREATED AT BAURU SCHOOL OF DENTISTRY, UNIVERSITY OF SAO PAULO
}

\author{
Milton Carlos Gonçalves SALVADOR ${ }^{1}$, Accácio Lins do VALLE ${ }^{2}$, \\ Mariana Carvalho Mandim RIBEIRO ${ }^{3}$, Jefferson Ricardo PEREIRA ${ }^{4}$
}

1- DDS, PhD, Professor, Department of Prosthodontics, Bauru School of Dentistry, University of Sao Paulo, Brazil.

2- DDS, PhD, Associated Professor, Department of Prosthodontics, Bauru School of Dentistry, University of Sao Paulo, Brazil.

3- DDS, MSc, Department of Prosthodontics, Bauru School of Dentistry, University of Sao Paulo, Brazil.

4- DDS, MSc, Graduated student (Doctoral degree), Department of Prosthodontics, Bauru School of Dentistry, University of Sao Paulo, Brazil.

Corresponding address: Milton Carlos Gonçalves Salvador - Department of Prosthodontics - Al. Dr. Octávio Pinheiro Brisola, 9-75 - Bauru, Sao Paulo - Cep.: 17012-901 - Brazil - phone number: 5514 3235-8277

Received: September 27, 2004 - Modification: August 30, 2005 - Accepted: August 11, 2006

\begin{abstract}
A

group of destructive changes occurring in jaws in patients with maxillary complete dentures and mandibular removable partial dentures (bilaterally) has been described in the literature as the combination syndrome. However, this condition is not clinically observed in all patients. The aim of this study was to establish the prevalence index on signs of combination syndrome and to verify whether these changes also occurred in patients rehabilitated with a mandibular removable partial denture (unilaterally). Sample was composed of 44 patients, completely edentulous in the maxilla. Thirty-two patients had a Kennedy Class I removable partial denture and 12 a Kennedy Class II. Three major alterations were observed in $20.5 \%$ of the studied population. Nevertheless, these changes were present only in $25 \%$ of patients with Kennedy Class I removable partial denture. Based on the findings of this study, it can be concluded that patients with Kennedy Class II removable partial denture do not have similar signs that lead to the combination syndrome's condition.

Uniterms: Combination syndrome; Kelly syndrome; Removable partial denture; Complete denture.
\end{abstract}

\section{INTRODUCTION}

Treatment of partially edentulous patients with absence of posterior teeth is a complex problem, manly due to its prognosis. Although improvements on the design and the material used to cast removable partial dentures (RPDs) have been made, resorption of the edentulous ridges and secondary alterations on the surrounding soft tissues still are factors difficult to control.

Light and intermittent applied forces can stimulate and preserve residual ridges. Even with maximum coverage of support bearing maxillary and mandibular areas, occlusal instability may result from wearing of artificial teeth along with resorption of the underlying bone. Changes in the Vertical Dimension of Occlusion lead to bite with anterior teeth, overloading the anterior portion of the edentulous maxilla.

This situation becomes more aggressive when a maxillary complete denture opposes a mandibular removable partial denture (RPD). Kelly ${ }^{9}$, in 1972, from clinical observations, verified the presence of some of these clinical signs: (1) loss of bone from the anterior part of the maxillary ridge, (2) overgrowth of the tuberosities, (3) papillary hyperplasia in the hard palate, (4) extrusion of the mandibular anterior teeth and (5) loss of bone under the partial denture bases. These changes were known as "combination syndrome". Later in 1979, Saunders, et al. ${ }^{14}$ added six more characteristics to the Kelly's ${ }^{9}$ work: (1) loss of vertical dimension of occlusion, (2) occlusal plane discrepancy, (3) anterior spatial repositioning of the mandible, (4) poor adaptation of the prostheses, (5) epulis fissuratum and (6) periodontal changes.

The changes in tissue form and health seen in this syndrome can be attributed to several factors, one of which is the biomechanical factor. When mandibular anterior teeth are present, patients tend to favor these teeth functionally because of the ability to generate maximum force. Excessive anterior function and parafunction in excursive movements constantly overload the anterior ridge to result in alveolar bone resorption and possible development of epulis fissuratum. As bone and ridge height are lost anteriorly, tuberosities in the posterior region will often enlarge and grow downward. One theory suggests that negative pressure within the maxillary denture pulls the tuberosities down as the anterior ridge is driven upward by the anterior occlusion? ${ }^{9}$. 
The functional load will then direct stress to the mandibular distal extension and cause more bone resorption of the mandibular ridge. The upward tipping movement of the anterior portion of the maxillary denture and the simultaneous downward movement of the posterior portion will decrease antagonist forces on the mandibular anterior teeth and lead to their supraeruption. Eventually, an occlusal plane discrepancy will occur and the patient may have a loss of vertical dimension of occlusion. In addition, the chronic stress and movement of the denture will often result in an ill-fitting prosthesis and contribute to the formation of palatal papillary hyperplasia.

According to Kelly ${ }^{9}$, rehabilitation of this type of patient is a common occurrence. He believes that these patients represent about $26 \%$ of individuals rehabilitated with complete prostheses. Of this percentage only $24 \%$ will develop these alterations ${ }^{9,15}$ and under different levels ${ }^{9}$.

The purpose of this study was to verify whether patients with a Kennedy Class II mandibular removable partial denture could present a similar condition to the combination syndrome and the prevalence index on signs of combination syndrome in patients with a Kennedy Class I or II RPD, treated at Bauru School of Dentistry, University of Sao Paulo. The null hypothesis of the present study was that there was not a similar condition to the combination syndrome in patients rehabilitated with a Kennedy Class II mandibular removable partial denture.

\section{MATERIALAND METHODS}

Completely edentulous patients in maxillary arches were randomly selected from the patients' records at the Department of Prosthodontics, Bauru School of Dentistry, University of Sao Paulo, Brazil. Criteria for selection were patients rehabilitated with a conventional complete maxillary denture and a mandibular removable partial denture (Kennedy class I or Kennedy class II). Further criteria were that they had been using the prostheses for not less than 2 years. Patients with presence of parafunctional occlusal forces and with a history of systemic disease that could affect bone metabolism or accelerate the resorption process were excluded.

The study protocol was approved by the institutional ethics committee of University of Sao Paulo - Bauru, and the subjects were enrolled in the study after signing the informed consent.

The final sample consisted of 44 subjects who were treated according to the policy at the department. It can therefore be assumed that posterior bilateral contact and well fitting prostheses were present at the time of placement. Patients were allocated in two groups: group one consisted of 32 patients (73\%) (Kennedy Class I) and group two of 12 patients (27\%) (Kennedy Class II). Mean time use of RPDs for group one was 5 years and for group two, 4.5 years. Mean time use of complete prostheses for group one was 8.5 years and for group two, 8 years.

\section{Clinical examination}

Some clinical aspects of the combination syndrome were assessed by one calibrated operator, including (see some examples in figures 1, 2, 3 and 4) (1) hypermobility of the anterior part of the maxilla; the anterior maxillary residual was palpated looking for a presence of loose hypermobile tissue overlying the alveolar ridge; (2) growth of the tuberosities; (3) papillary hyperplasia; (4) extrusion of the mandibular anterior teeth; (5) epulis fissuratum; (6) lack of adaptation (maxillary and/or mandibular prostheses); (7) necessity for replacements (maxillary and/or mandibular prostheses). Only consistent signs were considered.

The prostheses were checked for stability and retention for each subject using conventional procedures for complete dentures and removable partial denture, and results were recorded as adequate or poor.

Adequate retention: resistance to vertical pull, and sufficient resistance to lateral forces.

Poor retention: no resistance to vertical pull and lateral forces, the prosthesis falls out of place.

Adequate stability: slight or no rocking on prostheses supporting structures when under pressure.

Poor stability: extreme rocking on supporting structures under pressure.

The prostheses were considered lack of adaptation if only one result was unfavorable (poor retention or poor stability). With regard to the necessity of their replacement, two unfavorable results had been considered (poor retention and poor stability), jointly with the subjective sense of the operator.

\section{Statistical analysis}

The data were analyzed with a Chi-Square test to determine the overall association among the variables of group 1 (Kennedy Class I) and group II (Kennedy Class I) and the overall variability within the test groups $(\hat{a}=.05)$.

After that, the prevalence index of combination syndrome was verified in all patients (44 patients), and finally the ChiSquare was used test to verify the prevalence of combination syndrome.

Patients with combination syndrome were considered when there were three or more significant signs. The prevalence of combination syndrome was verified on the three following situations:

I) - Situation 1, the patients should present:

- Hypermobile tissue overlying the alveolar ridge

- Growth of the tuberosities

- Extrusion of the mandibular anterior teeth

II) - Situation 2:

- Hypermobile tissue overlying the alveolar ridge

- Growth of the tuberosities

- Extrusion of the mandibular anterior teeth

Papillary hyperplasia in the hard palate

III - Situation 3:

- Hypermobile tissue overlying the alveolar ridge

- Growth of the tuberosities

Extrusion of the mandibular anterior teeth 
Papillary hyperplasia in the hard palate - Inflammatory hiperplasia

The confidence level was $95 \%$.

\section{RESULTS}

Chi-Square test did not verify association between mandibular arch classification (Kennedy Class I and II) and all studied factors ( $\mathrm{p}>0.05)$

Regarding to lack of adaptation, group 2 showed a prevalence index of $60 \%$ against $33 \%$ in group 1 . The growth of the tuberosities presented similar prevalence indexes for both groups, although this was not expected in group 2 . With respect to the hypermobility, group 1 had a prevalence index of $81 \%$ and group 2 of $75 \%$.

From the original forty four patients, only nine $(20.5 \%)$ presented with the following characteristics: hypermobility, growth of the tuberosities and extrusion of mandibular anterior teeth. When two extra signs were added up (papillary hyperplasia and epulis fissuratum), the prevalence index was $13.6 \%$ (6 patients) and $2.3 \%$ (1 patient), respectively.

In group 1 , from 32 patients, only 8 (25\%) simultaneously presented the following signs: hypermobility, growth of the tuberosities and extrusion of the mandibular anterior teeth. In group 2 only one patient $(8 \%)$ had concomitantly the

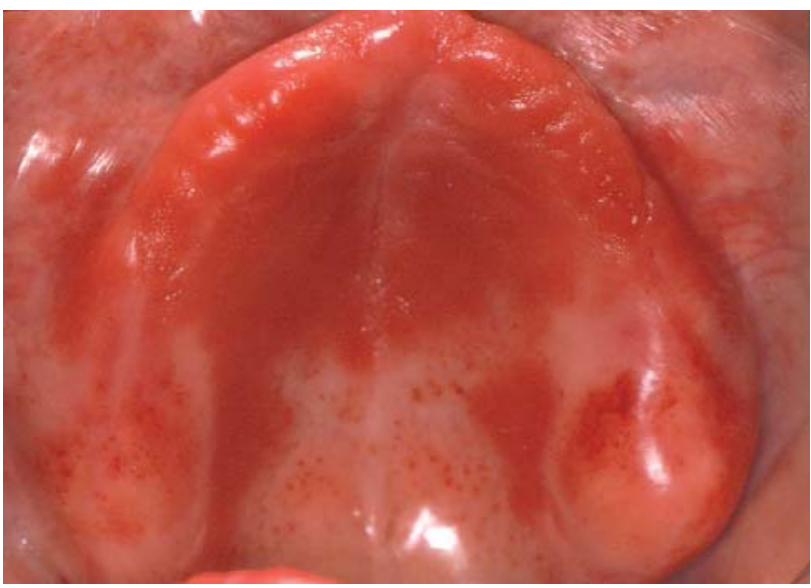

FIGURE 1- Overgrowth of the tuberosities

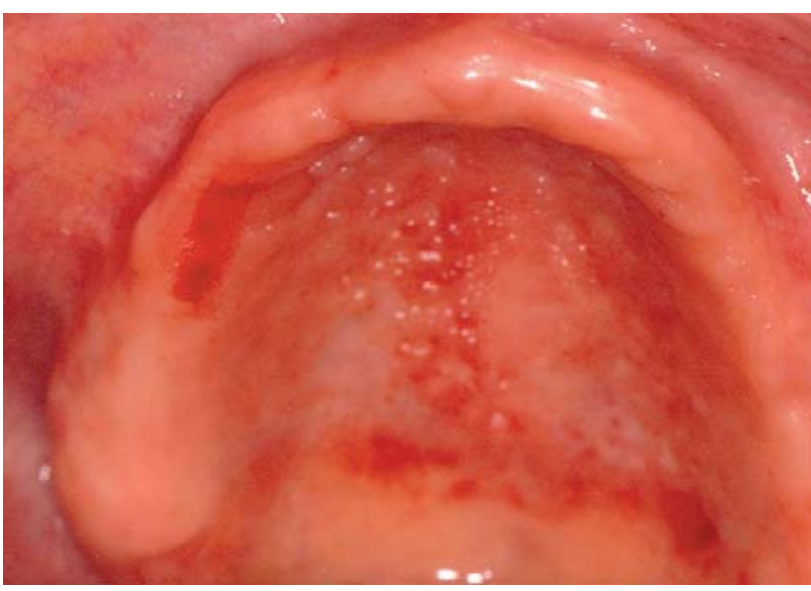

FIGURE 2- Papillary hyperplasia in the hard palate same signs.

Adding one more characteristic (papillary hyperplasia) to the group 1 and 2, the prevalence index was $15.6 \%$ (5 patients) and $8 \%$ (one patient), respectively. The presence of epulis fissuratum in combination with the other characteristics provides a prevalence index of $3 \%$ in group 1 , without occurrences in group 2.

In none of these situations was a statistically significant association observed between combination syndrome and the presence of all characteristics at the same time (ChiSquare Test, $\mathrm{p}>0.05$ ).

\section{DISCUSSION}

Based on the biomechanical assumptions made by Kelly ${ }^{9}$ and Saunders, et al. ${ }^{14}$, a developing sequence could be expected through the years in patients with combination syndrome. However, lack of controlled longitudinal studies impairs analysis of data collected in this work, due to the reduced sample size and short observation periods.

This work did not find a detailed sequence of events, as would be expected. When prevalence indices were separated according to Kennedy's classification, the following values were summarized (Table 1).

When the signs were grouped together into three

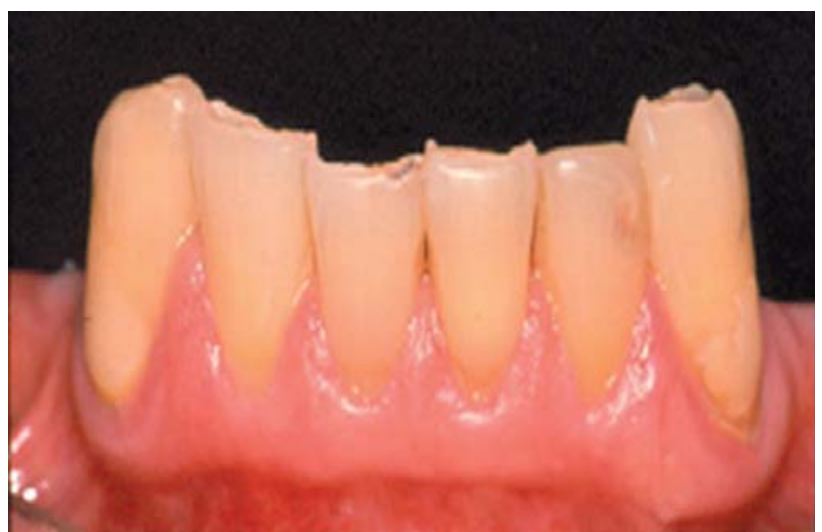

FIGURE 3- Extrusion of the mandibular anterior teeth

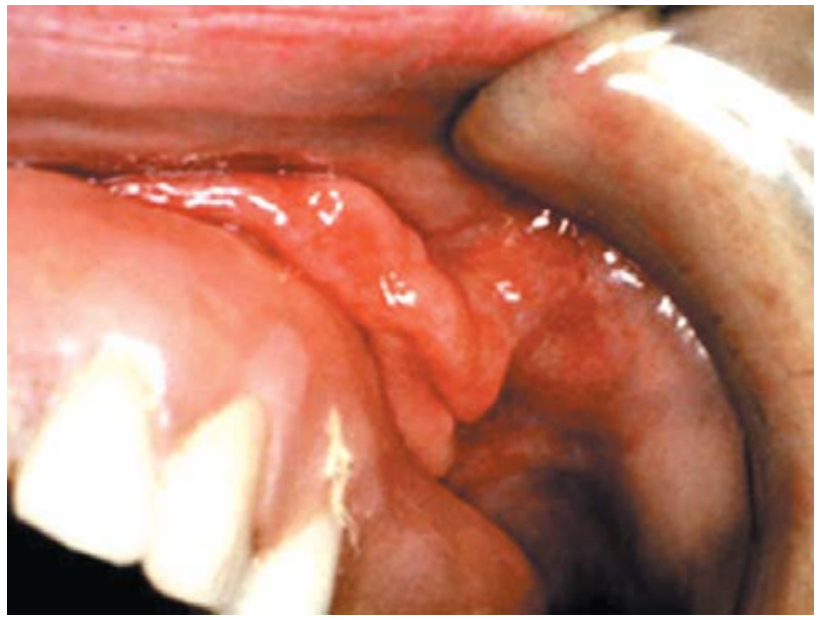

FIGURE 4- Epulis fissuratum 
possible situations, the following data could be generated (Table 2)

In the Class I situation, hypermobility, growth of the tuberosities and extrusion of mandibular anterior teeth provided the highest prevalence indices. In the Class II situation, hypermobility, growth of the tuberosities and epulis fissuratum were responsible for the same (Table 1).

Patients in group 1 had similar indices for resorption of the anterior ridge and papillary hyperplasia $(72.0 \%$ and 11.0 $\%$ ) and similar indices for lack of adaptation and necessity for replacement $(27.77 \%$ and $38.88 \%$, respectively). This confirms that resorption is a slow and gradual process, given that the mean use time of complete prosthesis was 9 years. Similarly, lack of adaptation and necessity for replacement showed that in a short period of time (5 years), lack of occlusal stability with acrylic teeth leads to a forward position of the mandible.

The growth of the tuberosities (44\%) is a characteristic that accentuates the lack of adaptation of the mandibular RPDs, leading to the periodic relining of dentures. Unfortunately, the design of this study does not permit enough follow-up time, not allowing establishment of a sequential pattern.

In group II, prevalence indices regarding to the lack of adaptation and necessity for replacement are even greater (range from 40 to $80 \%$, respectively) than in group 1 . The necessity for replacement of complete prosthesis has a greater prevalence than the necessity to replace the RPD. Maybe this can be explained by mechanical forces acting in a Kennedy Class II RPD, which generates less torque to abutment teeth. The growth of the tuberosities was often seen unilaterally, accentuating the lack of occlusal stability provided by acrylic teeth. One can theorize that disocclusion on the working side with natural teeth would generate a lever force on the non-working side with acrylic teeth, dislodging the complete prosthesis, giving space for down growth of the tuberosities and leading to the resorption of mandibular residual ridges. However, neither the functional occlusal scheme (canine guidance, group function) nor the type of occlusal stability (contacts between opposing acrylic or metallic surfaces) were analyzed. Hardness differences between acrylic and natural teeth would provide a more accentuated wear on the chewing preference side (natural teeth $)^{18}$.

The values for hypermobility and epulis fissuratum $(60 \%$ and $60 \%$ ) (Table 1) pointed out to the fact that a Class II situation can be more harmful than a Class I. However, this can not be stated since sample size is not weighted.

When all the characteristics were grouped together, it was observed that the higher the number of signs, the lower the prevalence index of combination syndrome, confirming the findings of Kelly ${ }^{9}$, Atwood ${ }^{1}$ and Tallgren ${ }^{16,17}$, that every patient has an individual reaction pattern to non-physiologic loads applied to the cortical and cancellous bone. In group 1 , prevalence index for combination syndrome was $16.66 \%$ and in group 2, prevalence was null. However, a lower prevalence index overlooks the necessity for periodical relining or replacement, according to the statements of Jackson, Ralph ${ }^{8}$.

At this time, it would be worthy to verify the connection between the clinical characteristics found and the satisfaction degree of patients with Class II RPDs, whereas more information could be added to search possible causes for the lack of adaptation. Literature has shown that the satisfaction degree is elevated when retention and stability are improved ${ }^{10,12}$. Not all patients are able to cope with removable dentures and some need additional retention (osseointegrated implants).

It would not be surprising to find some of the signs of combination syndrome in patients with osseointegrated implants. Some clinical studies ${ }^{12,15}$ have shown that bone loss is independent of the prosthesis type and others ${ }^{20}$ prove that overdentures can decrease the absorption rate in edentulous ridges.

Nevertheless, it would be desirable for all patients to incorporate a masticatory cycle, not to improve the chewing efficacy, but to minimize stress to the underlying structures. However, food crushing can only be done at the posterior area when there is an occlusal stability that provides adequate vertical dimension and sufficient space for

TABLE 2 - Prevalence indices grouped together into three possible situations

\begin{tabular}{lccc}
\hline & Situation 1 & Situation 2 & Situation 3 \\
\hline Class I & $16.66 \%$ & $5.55 \%$ & $5.55 \%$ \\
Class II & 0 & 0 & 0
\end{tabular}

TABLE 1- Prevalence indices individually separated according to type of RPD

\begin{tabular}{|c|c|c|c|c|c|c|c|c|c|}
\hline & ELAT & EF & GT & HAM & $\mathrm{PH}$ & $\begin{array}{c}\text { NR } \\
\text { maxillary }\end{array}$ & $\begin{array}{c}\text { NR } \\
\text { mandibular }\end{array}$ & $\begin{array}{c}\text { LA } \\
\text { maxillary }\end{array}$ & $\begin{array}{c}\text { LA } \\
\text { mandibular }\end{array}$ \\
\hline $\mathrm{ClI}$ & 16.66 & 5.50 & 44.00 & 72.0 & 11.0 & 38.88 & 33.33 & 27.77 & 27.77 \\
\hline $\mathrm{Cl} I \mathrm{I}$ & 0 & 60.0 & 40.00 & 60.0 & 20.0 & 80.0 & 40.00 & 60.00 & 60.00 \\
\hline
\end{tabular}

RPD: removable partial denture; ELAT: extrusion of the lower anterior teeth; EF: epulis fissuratum; GT: growth of the tuberosities; HAM: hypermobility of anterior part of the maxillae; $\mathrm{PH}$ : papillary hyperplasia; NR: necessity for replacements; LA: lack of adaptation. 
swallowing. How is it possible to correct the forward movement of the mandible if the syndrome manifestations have an individual pattern? Maybe osseointegrated implants would provide long term responses.

In accordance with the literature, the bone resorption pattern was not related with gender or age $\mathrm{e}^{1,16,17}$.

The mean time use of RPDs was 5 years and for complete prosthesis it was 8 years. The clinical exam revealed that 30 to $40 \%$ of prostheses need to be replaced or have lack of adaptation. The necessity to replace in group 2 is greater than in group 1 and perhaps due to the unbalanced sample size (5 patients in group 2 and 18 patients in group 1). Although this work had collected important information, one may think whether periodic relining would diminish the necessity for replacement or not.

As observed in the literature, the overall prevalence index for combination syndrome is low. However, one has to bear in mind that each patient has an individual response pattern to masticatory forces. Once installed, resorption will lead to a slow and gradual bone loss. Osseointegrated implants would minimize discomfort and maybe postpone the survival of the prosthesis $2,4,5,7,21$.

\section{CONCLUSIONS}

Considering the limitations of this study, the following conclusions can be drawn:

1. Combination syndrome was not observed in patients with complete prosthesis and Kennedy Class II RPDs;

2. Overall prevalence index for combination syndrome was $25 \%$.

\section{REFERENCES}

1 - Atwood DA. Reduction of the residual ridges: A major oral disease entity. J Prosthet Dent. 1971;26:266-79

2- Burns DR, Unger JW, Elsewick RK Jr, Giglio JA. Prospective clinical evaluation of mandibular implant overdentures. Part II: patient satisfaction and preference. J Prosthet Dent. 1995;73:364-9.

3- Consolaro A. Reabsorções dentárias nas especialidades clínicas. Maringá: Dental Press; 2002.

4- Crum RJ, Rooney GE. Alveolar bone loss in overdentures: A 5 year study. J Prosthet Dent. 1978;40:610-3.

5 - Cune MS, De Putter C, Hoogstraten J. Characteristics of 5410 edentulous implants candidates and the treatment they receive. Comm Dent Oral Epidemiol. 1995;23:110-3.

6- Douglass JB, Meader L, Kaplan A, Ellinger CW. Cephalometric evaluation of the changes in patients wearing complete dentures. A 20-year study. J Prosthet Dent. 1993;69:270-5.

7- Geertman ME, van Waas MA, van't Hof MA, Kalk W. Denture satisfaction in a comparative study of implant-retained mandibular overdentures: a randomized clinical trial. Int J Oral Maxillofac Implants. 1996;11:194-200.

8 - Jackson RA, Ralph WJ. Continuing changes in the contour of the maxillary residual alveolar ridge. J Oral Rehabil. 1980;7:245-8.
9- Kelly E. Changes caused by a mandibular removable partial denture opposing a maxillary complete denture. J Prosthet Dent. 1972;27:140-50

10 - Lechner SK, Mammen A. Combination syndrome in relation to osseointegrated implant-supported overdentures: a survey. Int J Prosthodont. 1996;9:58-64.

11 - Maxson BB, Powers MP, Scott RF. Prosthodontic considerations for the transmandibular implant. J Prosthet Dent. 1998;63:554-8.

12- Narhi TO, Geertman ME, Hevinga M, Abdo H, Kalk W. Changes in the edentulous maxilla in persons wearing implant-retained mandibular overdentures. J Prosthet Dent. 2000;84:43-9.

13- Salonen MA. Assessment of states of dentures and interest in implant-retained prosthetic treatment in 55 year edentulous Finns. Comm Dent Oral Epidemiol. 1994;22:130-5.

14- Saunders TR, Gillis RE, Desjardins RP. The maxillary complete denture opposing the mandibular bilateral distal-extension partial denture: treatment considerations. J Prosthet Dent. 1979;41:124-8.

15- Shen K, Gongloff RK. Prevalence of the 'combination syndrome' among denture patients. J Prosthet Dent. 1989;62:642-4.

16- Tallgren A. The continuing reduction of residual alveolar ridges incomplete denture wearers: a mixed - longitudinal study covering 25 years. J Prosthet Dent. 1972;27:120-32.

17- Tallgren A, Tryde G. Relationships between facial morphology and activity of orofacial muscles in patients with a complete upper and a partial lower denture. J Oral Rehabil. 1995;22:643-51.

18- Thiel CP, Evans DB, Burnett RR. Combination syndrome associated with a mandibular implant-supported overdenture: a clinical report. J Prosthet Dent. 1996;75:107-13.

19- Toolson LB, Taylor TD. A ten-year report of a longitudinal recall of overdentures patients. J Prosthet Dent. 1989;62:179-81.

20- Wyatt CC. The effect of prosthodontic treatment on alveolar bone loss: a review of the literature. J Prosthet Dent. 1998;80:3626.

21- Zarb GA, Bolender CL, Carlsson GN. Boucher's prosthodontic treatment for edentulous patients. Chicago: Mosby; 1997. 